\title{
THE ASYMPTOTIC DISTRIBUTION OF THE EIGENVALUES OF MULTIPARAMETER STURM-LIOUVILLE SYSTEMS II
}

\author{
by BRYAN P. RYNNE
}

(Received 22nd September 1992)

\begin{abstract}
In a previous paper we studied the asymptotic distribution of the multiparameter eigenvalues of uniformly right definite multiparameter Sturm-Liouville eigenvalue problems. In this paper we extend the analysis to deal with multiparameter Sturm-Liouville problems satisfying uniform left definiteness, and non-uniform left and right definiteness.
\end{abstract}

1980 Mathematics subject classification: 34L20, 34B24.

\section{Introduction}

Consider the $k$-parameter Sturm-Liouville eigenvalue problem

$$
\begin{gathered}
u_{r}^{\prime \prime}\left(x_{r}\right)+\left(q_{r}\left(x_{r}\right)+\sum_{s=1}^{k} \lambda_{s} v_{r s}\left(x_{r}\right)\right) u_{r}\left(x_{r}\right)=0, \quad 0 \leqq x_{r} \leqq 1, \quad r=1, \ldots, k, \\
u_{r}(0) \cos \alpha_{r}-u_{r}^{\prime}(0) \sin \alpha_{r}=0, \quad u_{r}(1) \cos \beta_{r}-u_{r}^{\prime}(1) \sin \beta_{r}=0, \quad r=1, \ldots, k,
\end{gathered}
$$

where $q_{r}, r=1, \ldots, k$, are real valued, continuous functions on the interval $U=[0,1], v_{r s}$, $r, s=1, \ldots, k$, are real valued, twice continuously differentiable $\left(C^{2}\right)$ functions on $U$ and $\alpha_{r}, \beta_{r} \in[0,2 \pi]$. A $k$-tuple $\lambda=\left(\lambda_{1}, \ldots, \lambda_{k}\right)$ of real numbers is called an eigenvalue of (1.1), $(1,2)$ if, for each $r$, there exists a non-trivial solution $u_{r}$ of equation (1.1) satisfying the boundary conditions (1.2).

For all $\mathbf{x}=\left(x_{1}, \ldots, x_{k}\right) \in U^{k}$ we define the determinant

$$
\Delta(\mathbf{x})=\underset{1 \leqq r, s \leqq k}{\operatorname{det}} v_{r s}\left(x_{r}\right) .
$$

The multiparameter eigenvalue problem (1.1), (1.2) is said to be uniformly right definite if $\Delta(x)>0$ for all $x \in U^{k}$, and is said to be right definite if $\Delta(x)>0$ for almost all $x \in U^{k}$. If the system (1.1), (1.2) is right definite then the basic result regarding the existence of eigenvalues is Klein's oscillation theorem (see [1] or [9] for the case of uniform right definiteness, and [4] for right definiteness);

Theorem 1.1. For each multi-index $\mathrm{i}=\left(i_{1}, \ldots, i_{k}\right)$ where $i_{1}, \ldots, i_{k}$, are non-negative 
integers, there exists a unique eigenvalue $\lambda^{i}$ of (1.1), (1.2) such that, for each $r, a$ corresponding solution of (1.1), (1.2) has precisely $i_{r}$ zeros in the open interval $(0,1)$. There are no other eigenvalues.

In [10] we studied the asymptotic behaviour of the eigenvalues $\lambda^{i}$ of $(1.1),(1.2)$, for large $\|\mathbf{i}\|$ (where $\|\cdot\|$ denotes the Euclidean norm in $\mathbf{R}^{k}$ ), and we showed that if the system is uniformly right definite then the eigenvalues have the asymptotic form

$$
\lambda^{i}=\pi^{2}\|i\|^{2} \psi(i /\|i\|)+O\left(\|i\|^{4 / 3}\right),
$$

where $\psi: \overline{\mathbf{R}}_{+}^{k} \cap S_{1} \rightarrow \mathbf{R}^{k}$ is Hölder continuous and non-zero on $\tilde{\mathbf{R}}_{+}^{k} \cap S_{1}$ $\left(\mathbf{R}_{ \pm}=\{x \in \mathbf{R}: \pm x>0\}, S_{1}=\left\{\mathbf{x} \in \mathbf{R}^{k}:\|\mathbf{x}\|=1\right\}\right)$. Conditions were also given which made $\psi$ Lipschitz continuous and reduced the size of the error term in (1.3) to $O(\|\mathrm{i}\|)$, see Theorem 3.1 of [10]. In this paper we investigate the asymptotic behaviour of the eigenvalues under other definiteness conditions and discuss to what extent the behaviour described by (1.3) is preserved. Specifically, we consider uniform left definiteness and (non-uniform) right and left definiteness (each of these conditions are sufficient to ensure that an analogue of Klein's theorem is valid, see [5]).

The asymptotic behaviour of the eigenvalues has not been considered before under conditions other than uniform right definiteness. In the case where $k=2$ and the system is uniformly right definite this problem has been studied in great detail by Faierman in the papers [7], [8]. The two parameter case has also been studied recently by Browne and Sleeman in [6]. In [6] the asymptotic estimates given in [10] are improved for the case of those eigenvalues which lie in certain cones in the parameter space.

\section{Notation and preliminary results}

In this section we briefly recall some notation and results from [10]. We begin with a basic result on the number of zeros of solutions of a Sturm-Liouville type differential equation which was proved in [10].

Hypothesis F. Suppose that the function $f: U \rightarrow \mathbf{R}$ is $C^{2}$ and the set $\{x \in U: f(x)>0\}$ can be decomposed into the union of a finite number of disjoint, open intervals $I_{i}=\left(a_{i}^{1}, a_{i}^{2}\right)$, $i=1, \ldots, n$, together with any of the end points $a_{i}^{j}$, which are not zeros of $f$, and there exists a constant $K>1$ such that on each interval $I_{i}$ either:

$$
\frac{\left|\left(x-a_{i}^{1}\right) f^{\prime}(x)\right|}{f(x)} \leqq K, \quad \frac{\left|\left(x-a_{i}^{1}\right)^{2} f^{\prime \prime}(x)\right|}{f(x)} \leqq K, \quad i=1, \ldots, n
$$

(ii) there is an increasing function $f_{i}$ such that,

$$
K^{-1} \widetilde{f}_{i}(x) \leqq f(x) \leqq K \widetilde{f}_{i}(x)
$$

or (i) holds with $a_{i}^{1}$ replaced by $a_{i}^{2}$, and (ii) holds with $f$ decreasing. 
Hypothesis UF. Suppose that the function $f: U \times X \rightarrow \mathbf{R}$ is such that for each $\xi \in X$ the function $f(\cdot, \xi): U \rightarrow \mathbf{R}$ satisfies hypothesis $F$, and let $n(\xi), K(\xi)$ denote the number of intervals and the constant in hypothesis $F$. Then $f$ is said to satisfy hypothesis $U F$ if $n(\xi)$ and $K(\xi)$ are uniformly bounded for $\xi \in X$, i.e. there exist constants $n>0, K>0$, such that

$$
n(\xi) \leqq n, \quad K(\xi) \leqq K, \quad \xi \in X .
$$

For any function $f: U \rightarrow \mathbf{R}$, let $\|f\|=\sup \{|f(x)|: x \in U\}$ and let $[f]_{+}$denote the function $x \rightarrow \max \{f(x), 0\}, x \in U$.

Lemma 2.1. Consider the differential equation

$$
w^{\prime \prime}(x)+p(x) w(x)+\mu f(x, \xi) w(x)=0, \quad x \in U, \quad \xi \in X,
$$

where $p$ is a real-valued, continuous function in $U$ and $f$ satisfies hypothesis $U F$. Then for all $\mu>0$, and $\xi \in X$, the number of zeros $v(w)$ of any solution $w$ of $(2.3)$ in the interval $(0,1)$ satisfies

$$
v(w)=\pi^{-1} \mu^{1 / 2} \int_{0}^{1}[f(x, \xi)]_{+}^{1 / 2} d x+O(1)
$$

where $|O(1)| \leqq 2(n+1)\left(\pi^{-1}\|p\|^{1 / 2}+\left(K^{2}+\|p\|\right) K^{2}+5\right)$.

To show that a significant class of functions $f$ satisfy hypothesis $U F$, the following lemma was proved in [10].

Lemma 2.2. Suppose that $X$ is a compact topological space and $A$ is a complex domain containing $U$, and suppose that $f: A \times X \rightarrow \mathbb{C}^{n}$ is continuous on $A \times X$ and, for each $\xi \in X$, the function $f(\cdot, \xi) \not \equiv 0$ is analytic on $A$. Also suppose that $\left.f\right|_{U \times X}$ (the restriction of $f$ to $U \times X)$ is real-valued. Then $\left.f\right|_{U \times X}$ satisfies hypothesis $U F$.

We now introduce some further notation. For any set $A$, we let $\bar{A}, \partial A$, int $A$ denote the closure, boundary and interior of $A$ respectively. Constants $c_{i}, i=1,2, \ldots$, will be strictly positive and may depend on $k$ and the functions $p_{r}, v_{r s}$, but will not depend on $\lambda$, or on any other variables. For any two quantities $a, b$, possibly depending on other variables, we will use the notation $a \approx b$ if there exist constants $c_{1}, c_{2}$, such that

$$
c_{1} \leqq \frac{a}{b} \leqq c_{2}
$$

For $\mathbf{a} \in \mathbf{R}^{k}$, we write $\mathbf{a}>\mathbf{0}$ (respectively $\mathbf{a} \geqq 0$ ) if, and only if, $a_{r}>0$ (respectively $a_{r} \geqq 0$ ), for all $r=1, \ldots, k$. For any real (or vector) valued function $f$, we use the notation $f>0$ (or $f>0$ ) to mean $f(x)>0$ (or $f(x)>0$ ) for all $x$ in the domain of $f$; the meaning of the notation $f \geqq 0(f \geqq 0), f \neq 0(f \neq 0)$, etc. is defined similarly. 
For each $r=1, \ldots, k$, we let $T_{r}: D\left(T_{r}\right) \subset L^{2}(U) \rightarrow L^{2}(U)$ denote the self-adjoint differential operator on $L^{2}(U)$ associated with the differential expression on the left-hand side of (1.1), with $\lambda=0$, together with the boundary conditions (1.2). Also, for $r=1, \ldots, k$, we define the functions

$$
\begin{gathered}
\mathbf{v}_{r}\left(x_{r}\right)=\left(v_{r 1}\left(x_{r}\right), \ldots, v_{r k}\left(x_{r}\right)\right), \quad \lambda \cdot \mathbf{v}_{r}\left(x_{r}\right)=\sum_{s=1}^{k} \lambda_{s} v_{r s}\left(x_{r}\right), \quad x_{r} \in U, \quad \lambda \in \mathbf{R}^{k}, \\
\phi_{r}(\lambda)=\int_{U}\left[\lambda \cdot v_{r}\left(x_{r}\right)\right]_{+}^{1 / 2} d x_{r}, \quad \phi(\lambda)=\left(\phi_{1}(\lambda), \ldots, \phi_{k}(\lambda)\right), \quad \lambda \in \mathbf{R}^{k} .
\end{gathered}
$$

Clearly, the function $\phi: \mathbf{R}^{k} \rightarrow \mathbf{R}^{k}$ is continuous and $\phi(\lambda) \geqq 0$. Also, for any real $c \geqq 0$,

$$
\phi(c \lambda)=c^{1 / 2} \phi(\lambda)
$$

Let $Q$ denote the set of points $\lambda$ for which $\phi(\lambda)>0$. It follows from (2.5) that the sets $Q$ and $\bar{Q}$ are cones (a set $A \subset \mathbf{R}^{k}$ is said to be a cone if, for any $\mathbf{a} \in A, c \mathbf{a} \in A$ for all $c>0$ ).

Throughout the paper we will assume that the functions $(x, \lambda) \rightarrow \lambda \cdot v_{r}(x),(x, \lambda) \in U \times \bar{Q}$, $r=1, \ldots, k$, satisfy hypothesis UF, so that the result of Lemma 2.1 holds for the differential equations (1.1).

\section{Radial behaviour of $\lambda^{i}$}

The estimate (1.3) shows that if the multiparameter system (1.1), (1.2) is uniformly right definite, then $\left\|\lambda^{i}\right\| \approx\|\mathbf{i}\|^{2}$ for large $\|\mathbf{i}\|$. In this section we consider whether this behaviour holds in general.

Suppose that, for some multi-index $i$, the eigenvalue $\lambda^{i}$ exists. By definition, for each $r=1, \ldots, k$, there is a solution of the differential equation (1.1) with $i_{r}$ zeros in $(0,1)$. Thus, by Lemma 2.1,

$$
\mathbf{i}=\pi^{-1} \phi\left(\lambda^{i}\right)+O(1)
$$

where the term $O(1)$ is bounded by a constant (given in Lemma 2.1) depending on the functions $p_{r}, v_{r s}$, but not on $\lambda$. Next, we observe that from the definition of $\phi$,

$$
\|\phi(\lambda)\|^{2} \leqq c_{3}\|\lambda\|, \quad \lambda \in \mathbf{R}^{k}
$$

Hence, if $\|\mathbf{i}\|$ is sufficiently large,

$$
\|\mathrm{i}\|^{2} \leqq c_{4}\left\|\lambda^{i}\right\|
$$

i.e. for any multiparameter problem of the form (1.1), (1.2), the eigenvalues $\lambda^{i}$ cannot grow more slowly than $\|\mathbf{i}\|^{2}$. 
Example. Consider the system

$$
\begin{gathered}
u_{1}^{\prime \prime}\left(x_{1}\right)+\lambda_{1} u_{1}\left(x_{1}\right)-\lambda_{2} x_{1}^{n} u_{1}\left(x_{1}\right)=0 \\
u_{2}^{\prime \prime}\left(x_{2}\right)+\lambda_{1} u_{2}\left(x_{2}\right)=0
\end{gathered}
$$

(where $n$ is any positive integer) with the boundary conditions

$$
u_{r}(0)=0, \quad u_{r}(1)=0, \quad r=1,2 .
$$

For this system the determinant $\Delta(\mathbf{x})=x_{1}^{n}$, so the system is right definite, but is not uniformly right definite.

We now study the behaviour of the eigenvalues $\lambda^{(1, m)}$ as $m \rightarrow \infty$. For a solution of (3.3) to have exactly $m$ interior zeros it is necessary that $\lambda_{1} \approx m^{2}$. For a solution of (3.2) to have exactly 1 interior zero it is necessary that $\phi_{1}(\lambda) \approx 1$. Now,

$$
\begin{aligned}
\phi_{1}(\lambda) & =\int_{0}^{\left(\lambda_{1} / \lambda_{2}\right)^{1 / n}}\left(\lambda_{1}-\lambda_{2} x_{1}^{n}\right)^{1 / 2} d x_{1}=\int_{0}^{1}\left(\lambda_{1}-\lambda_{1} z^{n}\right)^{1 / 2}\left(\lambda_{1} / \lambda_{2}\right)^{1 / n} d z \\
& =\frac{\lambda_{1}^{1 / 2+1 / n}}{\lambda_{2}^{1 / n}} \int_{0}^{1}\left(1-z^{n}\right)^{1 / 2} d z
\end{aligned}
$$

(substituting $x_{1}=\left(\lambda_{1} / \lambda_{2}\right)^{1 / n} z$ ). Therefore we must have $\lambda_{2} \approx \lambda_{1}^{n / 2+1} \approx m^{n+2}$, and so $\left\|\lambda^{(1, m)}\right\|$ $\approx m^{n+2}$.

This example shows that if we weaken uniform right definiteness to right definiteness then the eigenvalues $\lambda^{i}$ can grow faster than $\|i\|^{\alpha}$, for any positive $\alpha$. We will now describe a simple condition which ensures that $\left\|\lambda^{i}\right\| \approx\|\mathbf{i}\|^{2}$.

For each $r=1, \ldots, k$, let

$$
N_{r}=\left\{\lambda: \lambda \cdot v_{r} \leqq 0\right\}, \quad P_{r}=\left\{\lambda: \lambda \cdot v_{r} \geqq 0\right\} .
$$

The sets $N_{r}$ are cones and, for each $r, \mathbf{0} \in N_{r}$ and $\mathbf{0} \in \partial N_{r}$ unless $N_{r}=\mathbf{R}^{k}$, which only happens if $v_{r s} \equiv 0$ for all $s$.

Lemma 3.1. Suppose that the cones $N_{r}$ satisfy

$$
\bar{Q} \cap \bigcap_{r=1}^{k} \partial N_{r}=\{0\} .
$$

Then there exist constants $c_{5}, c_{6}>0$, such that for all $\lambda \in \bar{Q}$,

$$
c_{5}\|\lambda\| \leqq\|\phi(\lambda)\|^{2} \leqq c_{6}\|\lambda\| .
$$

Proof. We know that the second inequality holds in general, so we must prove the 
first inequality. Let $m_{r}(\lambda)=\max \left\{\lambda \cdot v_{r}\left(x_{r}\right): x_{r} \in U\right\}$. If $\lambda \in Q$, then for each $r, m_{r}(\lambda)>0$. Now suppose that there exists a sequence of unit vectors $\lambda^{n} \in Q, n=1,2, \ldots$, such that $m_{r}\left(\lambda^{n}\right) \rightarrow 0$, for all $r=1, \ldots, k$. By taking a subsequence, if necessary, we may suppose that $\lambda^{n} \rightarrow \lambda^{\infty} \in \bar{Q}, \lambda^{\infty} \neq 0$. Also, for each $r$ we have $m_{r}\left(\lambda^{n}\right)>0, n=1,2, \ldots$, but $m_{r}\left(\lambda^{\infty}\right)=0$ (by continuity), so $\lambda^{\infty} \in \partial N_{r}$, which, since $\lambda^{\infty} \neq 0$, contradicts (3.5). Thus there exists $c_{7}>0$ such that for any unit vector $\lambda \in \bar{Q}$ there is an $r$ for which $m_{r}(\lambda) \geqq c_{7}$. Hence, since the functions $v_{r s}$ are $C^{2}$, it follows that for any $\lambda \in \bar{Q}$, there is an $r$, and an interval $U_{\lambda} \subset U$ of length $\left|U_{\lambda}\right| \geqq c_{8}$, such that

$$
\lambda \cdot v_{r}\left(x_{r}\right) \geqq c_{9}\|\lambda\|, \quad x_{r} \in U_{\lambda},
$$

and so the result follows from the definition of $\phi$.

We observe that in the above example $\lambda=(0,1) \in \bar{Q} \cap \partial N_{1} \cap \partial N_{2}$, which is the direction of rapid growth of the eigenvalues $\lambda^{(1, m)}$.

Corollary 3.2. If (3.5) holds, then there exists a constant $c_{10}>0$ such that if the eigenvalue $\lambda^{i}$ exists and $\lambda^{i} \in \bar{Q}$, then

$$
\left\|\lambda^{\mathrm{i}}\right\| \leqq c_{10}\|\mathbf{i}\|^{2}
$$

We necessarily have $\lambda^{i} \in \bar{Q}$ if, for instance, the operators, $T_{\mathrm{r}}$ are negative definite (which is assumed in the left definite case, see Section 4). Thus, in this case (3.5) implies (3.7). Similarly, (3.5) implies (3.7) if the operators $T_{r}$ can be made negative definite by an eigenvalue transformation (as is the case under uniform right definiteness, for example).

The condition (3.5) in Lemma 3.1 does not depend on any particular definiteness conditions, but various such conditions ensure that it holds. For instance, it follows easily from Lemma 2.4 of [10] that uniform right definiteness implies (3.5). Also, it will be shown below that left definiteness (uniform and non-uniform) implies (3.5) (see Section 4 below for the definition of left definiteness and Sections 4 and 6 for the proof of this result). The above example shows that right definiteness does not imply (3.5); however, it will be shown in Section 5 that if the system is right definite and, for each $r$, the functions $\left\{v_{r s}: s=1, \ldots, k\right\}$ are linearly independent then (3.5) holds.

\section{The uniformly left definite case}

Let

$$
V(\mathbf{x})=\left(\begin{array}{ccc}
v_{11}\left(x_{1}\right) & \ldots & v_{1 k}\left(x_{1}\right) \\
\vdots & & \vdots \\
v_{k 1}\left(x_{k}\right) & \ldots & v_{k k}\left(x_{k}\right)
\end{array}\right), \mathrm{x} \in U^{k}
$$

The determinant $\Delta(\mathbf{x})=\operatorname{det} V(\mathbf{x})$, and we define $\Delta_{r s}(x)$ to be the cofactor of $v_{r s}\left(x_{r}\right)$ in the expansion of the determinant $\Delta(\mathbf{x})$. 
Definition. We say that the multiparameter system (1.1), (1.2) is uniformly left definite if the following two conditions hold:

$L D_{t}$ : each operator $T_{r}, r=1, \ldots, k$, is strictly negative definite.

$L D_{\delta}^{s}: \Delta_{r s}(\mathrm{x})>0$, for all $\mathrm{x} \in U^{k}, r, s=1, \ldots, k$.

We say that the multiparameter system (1.1), (1.2) is left definite if $L D_{t}$ and the following conditions holds:

$L D_{\delta}: \Delta_{r s}(\mathbf{x})>0$, for almost all $\mathrm{x} \in U^{k}, r, s=1, \ldots, k$.

Clearly a uniformly left definite system is left definite. Note that what we are calling uniform left definiteness has often been called simply left definiteness in the literature. Note also that conditions $L D_{\delta}^{s}$ is different to the condition used in the definition of (uniform) left definiteness in, for example, [11]; however, it is shown in [2] that the definitions are equivalent (after an invertible linear transformation of the eigenvalues, if necessary).

Suppose that (1.1), (1.2) is left definite. We define the cones $Q_{ \pm} \subset Q$ by

$$
Q_{ \pm}=\left\{\lambda \in \mathbf{R}^{k}: \exists \mathbf{x} \in U^{k} \text { such that } \lambda \cdot \mathbf{v}_{r}\left(x_{r}\right)>0, r=1, \ldots, k, \text { and } \pm \Delta(\mathbf{x})>0\right\} .
$$

The cones $Q_{ \pm}$are the analogues, in the present setting, of the cones $C_{ \pm}$defined in section 4.1 of [3] in an abstract setting. For any $\lambda \in Q$, there are open intervals $U_{r} \subset U$ such that $\lambda \cdot v_{r}\left(x_{r}\right)>0, x_{r} \in U_{r}, r=1, \ldots, k$, so, from $L D_{\delta}$, there is a point $\mathbf{x} \in U^{k}$ such that

$$
\Delta(\mathbf{x}) \lambda_{l}=\sum_{r, s=1}^{k} \Delta_{r l}(\mathbf{x}) v_{r s}\left(x_{r}\right) \lambda_{s}=\sum_{r=1}^{k} \Delta_{r l}(\mathbf{x}) \lambda \cdot \mathbf{v}_{r}\left(x_{r}\right)>0, \quad l=1, \ldots, k .
$$

It follows that $\Delta(\mathbf{x}) \neq 0$, so $\lambda \in Q_{-} \cup Q_{+}$and the components $\lambda_{l}, l=1, \ldots, k$, of $\lambda$ are non-zero and have the same sign as $\Delta(\mathbf{x})$. Thus, $Q=Q_{-} \cup Q_{+}$, and $Q_{ \pm} \subset \mathbf{R}_{ \pm}^{k}$.

In the left definite case we have the following Klein type oscillation theorem, see Corollary 5.6 of [5].

Theorem 4.1. Suppose that the multiparameter system (1.1), (1.2) is left definite. Then for each multi-index $\mathrm{i}$, and each non-empty cone $Q_{ \pm}$there exists a unique eigenvalue $\lambda^{i \pm} \in Q_{ \pm}$of (1.1), (1.2) such that, for each $r$, a corresponding solution of (1.1), (1.2) has precisely $i$, zeros in the open interval $(0,1)$. There are no other eigenvalues.

We remark that Corollary 5.6 of [5] uses a slightly weaker condition than $L D_{t}$, but condition $L D_{t}$ is required in the proofs of the main theorems below to ensure that the eigenvalues lie in the set $\bar{Q}$.

Note also that $Q_{ \pm} \neq \varnothing \Leftrightarrow \exists \mathbf{x} \in U^{k}$ such that $\pm \Delta(\mathbf{x})>0$. To avoid the trivial case of no eigenvalues we assume that $\Delta \neq 0$, and hence, by reordering the system if necessary, we may suppose that $\Delta(\mathbf{x})>0$ for some $x$, and so $Q_{+} \neq \varnothing$. We will discuss the distribution of the eigenvalues $\lambda^{i+}$ in $Q_{+} ;$a similar discussion applies to the $\lambda^{i-} \in Q_{-}$if $Q_{-} \neq \varnothing$.

For the remainder of this section we suppose that the system (1.1), (1.2) is uniformly left definite. The distribution of the eigenvalues in the (non-uniform) left definite case will be discussed in Section 6. 
We now define the matrices $V_{r}(\mathbf{x}), r=1, \ldots, k$, to be the matrices obtained by replacing the $r$ th row of $V(x)$ with the vector $\omega=(1, \ldots, 1)$. Condition $L D_{\delta}^{s}$ implies that for each $r$ and each $\mathbf{x} \in U^{k}$, det $V_{r}(\mathbf{x})>0$, so the matrix $V_{r}(\mathbf{x})$ is non-singular. Also, let $\omega^{\perp}$ denote the set $\left\{\lambda \in \mathbf{R}^{k}: \lambda \cdot \omega=0\right\}$.

Lemma 4.2. For any $v \in \omega^{\perp}$, there exist integers $r_{ \pm}$such that

$$
\pm v \cdot v_{r \pm} \geqq c_{11}\|v\| \text {. }
$$

Proof. Choose any unit vector $v \in \omega^{\perp}$. Now suppose that for some $r$, and all $r^{\prime} \neq r$, there exist points $x_{r^{\prime}}^{0}$ such that $v \cdot v_{r^{\prime}}\left(x_{r^{\prime}}^{0}\right)=0$. Then letting $\mathbf{x}^{0}=\left(x_{1}^{0}, \ldots, x_{k}^{0}\right)$, with $\mathbf{x}_{r}^{0}=\frac{1}{2}$ say, we have $V_{r}\left(\mathbf{x}^{0}\right) v=0$ (where $V_{r}\left(\mathbf{x}^{0}\right) v$ denotes the standard matrix product, with $v$ regarded as a column matrix), which contradicts the non-singularity of the matrix $V_{r}\left(x^{0}\right)$. Hence, for each $r$ there is an $r^{\prime} \neq r$ such that $v \cdot v_{r^{\prime}} \neq 0$; therefore, letting $R(v)$ denote the set of integers $r$ for which $v \cdot v_{r} \neq 0$, the number of elements of $R(v)$ is at least 2. Now suppose that $v \cdot v_{r}>0, r \in R(v)$. By the definition of $R(v)$, there exists a point $\mathrm{x} \in U^{k}$ such that

$$
v \cdot \mathbf{v}_{r}\left(x_{r}\right)=p_{r}, \quad r=1, \ldots, k,
$$

where $p_{r}>0, r \in R(v)$, and $p_{r}=0, r \notin R(v)$. From (4.1) and $L D_{\delta}^{s}$ we obtain

$$
\Delta(\mathbf{x}) v_{l}=\sum_{r=1}^{k} \Delta_{r l}(\mathbf{x}) \sum_{s=1}^{k} v_{r s}\left(x_{r}\right) v_{s}=\sum_{r=1}^{k} \Delta_{r l}(\mathbf{x}) p_{r}>0, \quad l=1, \ldots, k,
$$

so the numbers $v_{r}, r=1, \ldots, k$, are all non-zero and have the same sign. However, this contradicts the hypothesis that $v \cdot \omega=0$, and so our supposition that $v \cdot v_{r}>0, r \in R(v)$, must be false. Similarly, we cannot have $v \cdot v_{r}<0, r \in R(v)$. Therefore, letting $R_{ \pm}(v)$ denote the set of integers $r$ for which $\pm v \cdot v_{r}>0$, each set $R_{ \pm}(v)$ must be non-empty for any unit vector $v \in \omega^{\perp}$.

Now suppose that there exists a sequence of unit vectors $v^{n} \in \omega^{\perp}, n=1,2, \ldots$, such that for all $r \in R_{+}\left(v^{n}\right), \min \left\{v^{n} \cdot v_{r}\left(x_{r}\right): x_{r} \in U\right\}<n^{-1}$. By taking a subsequence, if necessary, we may assume that $v^{n} \rightarrow v^{\infty} \in \omega^{\perp}$, and the sets $R_{ \pm}\left(v^{n}\right), n=1,2, \ldots$, are constant, and equal to $R_{ \pm}$, say. By continuity, for each $r \in R_{+}$the function $v^{\infty} \cdot v_{r}$ has a zero, so $r \notin R_{+}\left(v^{\infty}\right)$; for each $r \in R_{-}$the function $v^{\infty} \cdot v_{r} \leqq 0$, so $r \notin R_{+}\left(v^{\infty}\right)$; for each $r \notin R_{+} \cup R_{-}$the function $v^{\infty} \cdot v_{r}$ has a zero, so $r \notin R_{+}\left(v^{\infty}\right)$. However, this contradicts the fact proved above that $R_{+}\left(v^{\infty}\right)$ must be non-empty. Therefore there exists a constant $c_{12}>0$ such that for all unit vectors $v \in \omega^{\perp}$, we have $v \cdot v_{r} \geqq c_{12}$, for some $r \in R_{+}(v)$. We can obtain a similar result for the set $R_{-}(v)$, so the lemma follows.

Lemma 4.3. If the system (1.1), (1.2) is uniformly left definite then condition (3.5) holds.

Proof. Suppose that $\lambda \neq 0$ and $\lambda \in \bar{Q} \cap \bigcap_{r=1}^{k} \partial N_{r}$. Since the set $Q=Q_{-} \cup Q_{+}$is a cone and $\bar{Q}_{ \pm} \subset \overline{\mathbf{R}}_{ \pm}^{k}$, there exists a vector $v \in \omega^{\perp}$ such that $\lambda+v \in Q$. However, since $\lambda \in \bigcap_{r=1}^{k}$ 
$\partial N_{r}$, we have $\lambda \cdot v_{r} \leqq 0$ for all $r$, and it follows from Lemma 4.2 that $v \cdot v_{r^{\prime}} \leqq 0$, for some $r^{\prime}$, hence $(\lambda+v) \cdot v_{r} \leqq 0$, which contradicts $\lambda+v \in Q$ and so proves the lemma.

The discussion on Section 3 now yields the following corollary.

Corollary 4.4. If the system (1.1), (1.2) is uniformly left definite then $\left\|\lambda^{i^{+}}\right\| \approx\|\mathrm{i}\|^{2}$, for all i. $A$ similar result holds for the eigenvalues $\lambda^{i-}$, if they exist.

Lemma 4.5. Suppose that $\lambda \in \bar{Q}_{+}$and $\|\lambda\|=1$. Then for any $\mu \in \mathbf{R}^{k}$ with $\|\boldsymbol{\mu}\| \leqq 1$ and $\lambda+\mu \in \bar{Q}_{+}$, we have:

$$
\|\phi(\lambda+\mu)-\phi(\lambda)\| \geqq c_{13}\|\mu\|^{3}
$$

(ii) if, for some $\varepsilon, 0<\varepsilon<1$, we have $\phi(\lambda) \geqq(\varepsilon, \ldots, \varepsilon)$ and $\phi(\lambda+\mu) \geqq(\varepsilon, \ldots, \varepsilon)$, then

$$
\|\phi(\lambda+\mu)-\phi(\lambda)\| \geqq \varepsilon c_{14}\|\mu\| .
$$

Proof. Since $\lambda \in \bar{Q}_{+} \subset \overline{\mathbf{R}}_{+}^{k}$ and $\omega=(1, \ldots, 1), \mu$ can be written in the form $\mu=\alpha \lambda+v$, where $v \in \omega^{\perp}$ and $\alpha, v$ are uniquely defined and satisfy

$$
c_{15}\|\mu\| \leqq|\alpha|+\|v\| \leqq c_{16}\|\mu\| .
$$

We may suppose that $\alpha \geqq 0$ (the case $\alpha<0$ can be dealt with similarly). Now suppose that $\alpha>c_{17}\|v\|^{1 / 2}$, where $c_{17}$ is sufficiently large (the specific criterion will be given below). Then, if $\phi_{r}(\lambda) \geqq c_{18}$ (this is true for some $r$ by Lemmas 3.1 and 4.3), we have

$$
\begin{aligned}
\phi_{r}(\lambda+\mu)-\phi_{r}(\lambda) & =\phi_{r}((1+\alpha) \lambda+v)-\phi_{r}(\lambda) \\
& \geqq \phi_{r}((1+\alpha) \lambda)-\phi_{r}(\lambda)-c_{19}\|v\|^{1 / 2} \\
& =\left((1+\alpha)^{1 / 2}-1\right) \phi_{r}(\lambda)-c_{19}\|v\|^{1 / 2} \\
& \geqq c_{20} \alpha \geqq c_{21}\|\mu\|,
\end{aligned}
$$

if $c_{17}$ is sufficiently large (the first inequality above is obtained from the integral defining $\phi_{r}((1+\alpha) \lambda+v)$ by applying the general inequality $[a+b]_{+}^{1 / 2} \geqq[a]_{+}^{1 / 2}-$ $2|b|^{1 / 2}, a, b \in \mathbf{R} ;$ the second inequality uses the fact that $\left.\alpha \leqq c_{16}\right)$.

Now suppose that $\alpha \leqq c_{17}\|v\|^{1 / 2}$, (and hence $\|\mu\| \leqq c_{22}\|v\|^{1 / 2}$, by (4.4), since $\|v\| \leqq c_{23}\|v\|^{1 / 2}$ ) and suppose that

$$
v \cdot v_{r} \geqq c_{24}\|v\|
$$

(this is true for some $r$ by Lemma 4.2). This inequality is (2.25) in the proof of Lemma 
2.6 of [10] (in the notation used there, we have $(1+\alpha) \lambda=\lambda^{1},(1+\alpha) \lambda+\nu=\lambda^{2}$ and $\left.v=\lambda^{2}-\lambda^{1}\right)$, and leads to inequality (2.31) in [10], which in the present case is

$$
\phi_{r}((1+\alpha) \lambda+v) \geqq \phi_{r}((1+\alpha) \lambda)+c_{2 s}\|v\|^{3 / 2} .
$$

Similarly, if the hypotheses of part (ii) of the lemma hold, inequality (2.31) in [10] leads to

$$
\phi_{r}((1+\alpha) \lambda+v) \geqq \phi_{r}((1+\alpha) \lambda)+c_{26} \varepsilon\|v\| .
$$

Thus, in the general case we have

$$
\begin{aligned}
\phi_{r}(\lambda+\mu)-\phi_{r}(\lambda) & =\phi_{r}((1+\alpha) \lambda+v)-\phi_{r}(\lambda) \\
& \geqq\left((1+\alpha)^{1 / 2}-1\right) \phi_{r}(\lambda)+c_{25}\|v\|^{3 / 2} \\
& \geqq c_{27}\|\mu\|^{3},
\end{aligned}
$$

and, if the hypotheses of part (ii) of the lemma hold, we have

$$
\begin{aligned}
\phi_{r}(\lambda+\mu)-\phi_{t}(\lambda) & =\phi_{r}((1+\alpha) \lambda+v)-\phi_{r}(\lambda) \\
& \geqq\left((1+\alpha)^{1 / 2}-1\right) \phi_{r}(\lambda)+c_{26} \varepsilon\|v\| \\
& \geqq c_{28} \varepsilon(\alpha+\|v\|) \geqq c_{29} \varepsilon\|\mu\| .
\end{aligned}
$$

These results prove the lemma.

We now let $\overline{\mathbf{R}}_{\varepsilon}^{k}$ denote the closed set in $\mathbf{R}^{k}$ consisting of those vectors $\lambda \neq 0$ such that $\lambda /\|\lambda\| \geqq(\varepsilon, \ldots, \varepsilon)$, together with 0 . Also, we let $\phi_{+}$denote the restriction of $\phi$ to the set $\bar{Q}_{+}$.

Theorem 4.6. The mapping $\phi_{+}: \bar{Q}_{+} \rightarrow \overline{\mathbf{R}}_{+}^{k}$ is a homeomorphism. Let $\phi_{+}^{-1}: \overline{\mathbf{R}}_{+}^{k} \rightarrow \bar{Q}_{+}$ denote the inverse of this homeomorphism. The restriction of $\phi_{+}^{-1}$ to $\overline{\mathbf{R}}_{+}^{k} \cap S_{1}$ is Hölder continuous with exponent $\frac{1}{3}$. The eigenvalues $\lambda^{i+}$ of the multiparameter problem satisfy

$$
\lambda^{i+}=\pi^{2} \phi_{+}^{-1}(\mathbf{i})+O\left(\|\mathbf{i}\|^{5 / 3}\right)=\pi^{2}\|\mathbf{i}\|^{2} \phi_{+}^{-1}(\mathbf{i} /\|\mathbf{i}\|)+O\left(\|\mathbf{i}\|^{5 / 3}\right)
$$

for all multi-indices $\mathbf{i} \neq \mathbf{0}$. For any $\varepsilon$ with $0<\varepsilon \leqq 1$, the restriction of $\phi_{+}^{-1}$ to $\overline{\mathbf{R}}_{\varepsilon}^{k} \cap S_{1}$ is Lipschitz continuous with Lipschitz constant $c_{30} \varepsilon^{-1}$, and for all $\mathbf{i} \in \overline{\mathbf{R}}_{\varepsilon}^{k}$,

$$
\lambda^{i+}=\pi^{2} \phi_{+}^{-1}(\mathbf{i})+\varepsilon^{-1} O(\|\mathrm{i}\|)=\pi^{2}\|\mathrm{i}\|^{2} \phi_{+}^{-1}(\mathrm{i} /\|\mathrm{i}\|)+\varepsilon^{-1} O(\|\mathrm{i}\|) .
$$


Proof. The proof is almost identical to the proof of Theorem 3.1 of [10], using the results of Lemma 4.5 .

We observe that the error estimate $O\left(\|i\|^{5 / 3}\right)$ is larger than the corresponding estimate $O\left(\|\mathrm{i}\|^{\mathbf{4} / 3}\right)$ obtained in Theorem 3.1 of [10]. This is due to the fact that we cannot assert that for all $\lambda \neq 0$ there is an $r$ such that $\lambda \cdot v_{r} \neq 0$, as we can under uniform right definiteness (c.f. Lemma 2.4 in [10] and Lemma 4.2 above). Probably both these estimates are too pessimistic for many multiparameter systems (in particular, see the third part of Theorem 3.1 of [10]).

\section{The right definite case}

In this section we suppose that the system (1.1), (1.2) is right definite. Lemma 3.1 gave a condition which ensures that the radial behaviour of the eigenvalues $\lambda^{i}$ is as described in (1.3) for large $\|\mathrm{i}\|$ (assuming, in addition, that condition $L D_{t}$ holds; see the discussion in Section 3). We will now discuss a condition which ensures that the 'angular' behaviour of the $\lambda^{i}$ is as described in (1.3), at least in subcones of $\bar{Q}$ which lie 'strictly' inside $Q$.

We begin with some constructions which will enable us to deal with the loss of uniformity compared with the uniform right definite case. For any $\mathbf{u}=\left(u_{1}, \ldots, u_{k}\right) \in$ $\bigoplus_{r=1}^{k} L^{2}(U)$, let $W(\mathbf{u})$ denote the $k \times k$ matrix with $(r, s)$ element equal to $\left(v_{r s} u_{r}, u_{r}\right)$, where $(\cdot, \cdot)$ denotes the inner product in $L^{2}(U)$. It can easily be seen that right definiteness implies that if $\mathrm{u}$ has $u_{\mathrm{r}} \neq 0, r=1, \ldots, k$, then $\operatorname{det} W(\mathrm{u}) \neq 0$, i.e. the matrix $W(\mathrm{u})$ is non-singular.

Lemma 5.1. For any $\lambda \in \mathbf{R}^{k}$ there exists $r$ such that $\lambda \in N_{r} \cup P_{r}$.

Proof. Suppose that there exists $\lambda \neq \mathbf{0}$ such that $\lambda \notin N_{\mathrm{r}} \cup P_{\mathrm{r}}$ for all $r=1, \ldots, k$ (i.e. the functions $\lambda \cdot v_{r}$ attain both positive and negative values). Then since the $r$ th element of the matrix product $W(\mathrm{u}) \lambda$ is given by

$$
\int_{0}^{1} \lambda \cdot v_{r}\left(x_{r}\right)\left|u_{r}\left(x_{r}\right)\right|^{2} d x_{r}
$$

we can choose non-zero functions $u_{r} \in L^{2}(U), r=1, \ldots, k$, such that $W(u) \lambda=0$, which contradicts right definiteness, and so proves the lemma.

Now, for each $r=1, \ldots, k$, let

$$
Z_{r}=\left\{\lambda \in \mathbf{R}^{k}: \lambda \cdot \mathbf{v}_{r} \equiv 0 \text { on a subset of } U \text { with positive measure }\right\}
$$

The set $Z_{r}$ need not be a subset of $N_{r} \cup P_{r}$, but if $Z_{r}$ intersects $N_{r} \cup P_{r}$ then $Z_{r} \cap\left(N_{r} \cup P_{r}\right) \subset \partial N_{r} \cup \partial P_{r}$ (to see this suppose that $\lambda \in Z_{r} \cap\left(N_{r} \cup P_{r}\right)$. Right definiteness implies that at least one of the functions $v_{r s}, s=1, \ldots, k$, is non-zero somewhere on the 
interval on which $\lambda \cdot v_{r} \equiv 0$, so there exist points $\lambda^{ \pm}$arbitrarily close to $\lambda$ for which $\left.\lambda^{+} \notin P_{r}, \lambda^{-} \notin N_{r}\right)$.

\section{Lemma 5.2. Suppose that}

$$
Z_{r} \cap\left(N_{r} \cup P_{r}\right) \subset\{0\} \cup \bigcup_{t=1}^{k}\left(\text { int } N_{t} \cup \operatorname{int} P_{t}\right), \quad r=1, \ldots, k
$$

Then, for any $\varepsilon$ with $0<\varepsilon \leqq 1$, there is a number $\gamma(\varepsilon)>0$ such that for any $\lambda \in \mathbf{R}^{k}$ there is an integer $r$, for which $\lambda \in N_{r} \cup P_{r}$ and

$$
\left|\lambda \cdot v_{r}\left(x_{r}\right)\right| \geqq \gamma(\varepsilon)|| \lambda \|, \quad x_{r} \notin U_{r}(\lambda, \varepsilon),
$$

where the set $U_{r}(\lambda, \varepsilon) \subset U$ has measure $\left|U_{r}(\lambda, \varepsilon)\right|<\varepsilon$.

Remark. If $Z_{r}=\{0\}, r=1, \ldots, k$ (e.g. if the functions $v_{r s}, s=1, \ldots, k$, are analytic and linearly independent for each $r$ ), then condition (5.1) is satisfied.

Proof. Suppose that the assertion of the lemma is false and there exists an $\varepsilon>0$ and a sequence of unit vectors $\lambda^{n}, n=1,2, \ldots$, such that, for those $r$ for which $\lambda^{n} \in N_{r} \cup P_{r}$, the sets

$$
U_{r}^{n}=\left\{x_{r} \in U:\left|\lambda^{n} \cdot \mathbf{v}_{r}\left(x_{r}\right)\right| \leqq 1 / n\right\} \subset U
$$

have measure $\left|U_{r}^{u}\right| \geqq \varepsilon$. By taking a subsequence, if necessary, we may assume that $\lambda^{n} \rightarrow \lambda^{\infty} \neq 0$, and that there is a set of integers $R$ such that $\lambda^{n} \in N_{r} \cup P_{r} \Leftrightarrow r \in R$, for all $n$. Letting $U_{r}^{\infty}=\bigcap_{N=1}^{\infty} \bigcup_{n=N} U_{r}^{n}, r \in R$, we have $\left|U_{r}^{\infty}\right| \geqq \varepsilon$ (see Q.2 in Exercises 13.2, p. 340 of [12]), and by continuity, $\lambda^{\infty} \cdot v_{r}\left(x_{r}\right)=0, x_{r} \in U_{r}^{\infty}$. Thus: for $r \in R$ we have $\lambda^{\infty} \in Z_{r}$, and so $\lambda^{\infty} \notin$ int $N_{r} \cup$ int $P_{r}\left(\right.$ since $\left.Z_{r} \cap\left(N_{r} \cup P_{r}\right) \subset \partial N_{r} \cup \partial P_{r}\right)$; for $r \notin R$ we have $\lambda^{\infty} \notin$ int $N_{r} \cup$ int $P_{r}$ (since $\lambda^{n} \notin N_{r} \cup P_{r}, n=1,2, \ldots$ ). This contradicts condition (5.1), and so complete the proof of the lemma.

Lemma 5.3. Suppose that (5.1) holds and that $0<\varepsilon<1$. There exists a number $\delta(\varepsilon)>0$ such that if $\lambda^{i} \in \bar{Q}_{+},\left\|\lambda^{i}\right\| \leqq 1, \phi\left(\lambda^{i}\right) \geqq(\varepsilon, \ldots, \varepsilon), i=1,2$, and $\left\|\lambda^{2}-\lambda^{1}\right\|$ is sufficiently small, then

$$
\left\|\phi\left(\lambda^{2}\right)-\phi\left(\lambda^{1}\right)\right\| \geqq \delta(\varepsilon)\left\|\lambda^{2}-\lambda^{1}\right\| .
$$

Proof. Let $\mu=\lambda^{2}-\lambda^{1}$. By definition, for any $r$,

$$
\phi_{r}\left(\lambda^{2}\right)-\phi_{r}\left(\lambda^{1}\right)=\int_{U} \delta_{r}\left(x_{r}\right) d x_{r}
$$

where $\delta_{r}\left(x_{r}\right)=\left[\lambda^{1} \cdot v_{r}\left(x_{r}\right)+\mu \cdot v_{r}\left(x_{r}\right)\right]_{+}^{1 / 2}-\left[\lambda^{1} \cdot v_{r}\left(x_{r}\right)\right]_{+}^{1 / 2}$. Now, the maximum of the func- 
tions $\lambda^{i} \cdot \mathbf{v}_{r}$ in $U$ is greater than $\varepsilon^{2}$, and since the functions $v_{r s}$ are $C^{2}$, it follows that $\lambda^{i} \cdot v_{r} \geqq \varepsilon^{2} / 2$ on an interval $U$, of length at least $2 c_{31} \varepsilon^{2}$. Also, by Lemma 5.2, there is an $r$ such that

$$
\mu \cdot v_{r}\left(x_{r}\right) \geqq \gamma\left(c_{31} \varepsilon^{2}\right)\|\mu\|, \quad x_{r} \notin U_{r}^{\prime},
$$

where $U_{r}^{\prime} \subset U$ is an interval with $\left|U_{r}^{\prime}\right|<c_{31} \varepsilon^{2}$ or $\left(\mu \cdot v_{r}(x) \leqq-\gamma\left(c_{31} \varepsilon^{2}\right)\|\mu\|, x \in U_{r}^{\prime}\right.$, in which case we interchange $\lambda^{1}$ and $\lambda^{2}$ to obtain (5.4)). Thus, the set $U_{r}^{\prime \prime}=U_{r} \cap\left(U \backslash U_{r}^{\prime}\right)$ has measure $\left|U_{r}^{\prime \prime}\right| \geqq c_{31} \varepsilon^{2}$, and

$$
\boldsymbol{\mu} \cdot \mathbf{v}_{r}\left(x_{r}\right) \geqq \gamma\left(c_{31} \varepsilon^{2}\right)\|\mu\|, \quad \lambda^{1} \cdot \mathbf{v}_{r}\left(x_{r}\right) \geqq \varepsilon^{2} / 2, \quad x_{r} \in U_{r}^{\prime \prime} .
$$

It follows from these inequalities that

$$
\delta_{r}\left(x_{r}\right) \geqq \frac{\gamma\left(c_{31} \varepsilon^{2}\right)\|\mu\|}{\left(\varepsilon^{2} / 2\right)^{1 / 2}+\left(\gamma\left(c_{31} \varepsilon^{2}\right)\|\mu\|\right)^{1 / 2}} \geqq \gamma\left(c_{31} \varepsilon^{2}\right)\|\mu\| \varepsilon^{-1}, \quad x_{r} \in U_{r}^{\prime \prime},
$$

if $\|\mu\|$ is sufficiently small. The inequality (5.2) now follows from this estimate, (5.3) and the estimate for $\left|U_{r}^{\prime \prime}\right|$.

Note that $\delta(\varepsilon)$ may tend to zero as $\varepsilon \rightarrow 0$.

Theorem 5.4. If (3.5) and (5.1) hold then the mapping $\phi: Q \rightarrow \mathbf{R}_{+}^{k}$ is a homeomorphism, with inverse $\phi^{-1}: \mathbf{R}_{+}^{k} \rightarrow Q$. Also, for any $\varepsilon$ with $0<\varepsilon \leqq 1$, the restriction of $\phi^{-1}$ to $\overline{\mathbf{R}}_{\varepsilon}^{k} \cap S_{1}$ is Lipschitz continuous with Lipschitz constant $\delta(\varepsilon)^{-1}$. If condition $L D_{t}$ also holds then, for all $\mathbf{i} \in \mathbf{R}_{\varepsilon}^{k}$,

$$
\lambda^{i}=\pi^{2} \phi^{-1}(\mathbf{i})+\delta(\varepsilon)^{-1} O(\|\mathbf{i}\|)=\pi^{2}\|\mathbf{i}\|^{2} \phi^{-1}(\mathbf{i} /\|\mathbf{i}\|)+\delta(\varepsilon)^{-1} O(\|\mathbf{i}\|) .
$$

Proof. The proof is almost identical to the proof of Theorem 3.1 of [10] using Lemmas 3.1 and 5.3 .

We will now give a simple criterion for condition (3.5) to be satisfied when the system is right definite.

Lemma 5.5. If, for each $r$, the functions $\left\{v_{r s}: s=1, \ldots, k\right\}$ are linearly independent then (3.5) holds.

Proof. Suppose that $\lambda \neq 0, \lambda \in \bar{Q} \cap \bigcap_{r=1}^{k} \partial N_{r}$, and let $\lambda^{n} \in Q, n=1,2, \ldots$, be a sequence of vectors such that $\lambda^{n} \rightarrow \lambda$. It follows from Lemma 5.1 that, after taking a subsequence if necessary, there is an $r$ such that $\lambda^{n} \in P_{r}$ for all $n$. But, by assumption, $\lambda \in \partial N_{r}$, so by continuity we must have $\lambda \cdot v_{r} \equiv 0$. However, this contradicts the linear independence of the functions $\left\{v_{r s}: s=1, \ldots, k\right\}$ and so proves the lemma. 
Remark. The conditions (3.5) and (5.1) are independent of each other in the sense that either can hold without the other. To see this let

$$
f_{0}(x)=\left\{\begin{array}{ll}
1-2 x, & 0 \leqq x \leqq \frac{1}{2}, \\
0, & \frac{1}{2} \leqq x \leqq 1,
\end{array} f_{1}(x)=1-x, \quad 0 \leqq x \leqq 1\right.
$$

and consider the $2 \times 2$ arrays of coefficient functions $v_{r s}$ given by

$$
\text { (i) }\left(\begin{array}{cc}
1 & -f_{1} \\
1 & f_{0}
\end{array}\right), \text { (ii) }\left(\begin{array}{cc}
1 & f_{1} \\
-1 & f_{1}
\end{array}\right) \text {. }
$$

It can be verified that both these arrays are right definite, but for (i), (3.5) holds and (5.1) does not, while for (ii), (3.5) does not hold and (5.1) does.

\section{The left definite case}

In this section we suppose that the multiparameter system (1.1), (1.2) is left definite as defined in Section 4. As noted in Section 4, the oscillation theorem, Theorem 4.1, is valid under this hypothesis. Again we will assume that $\Delta(x)>0$ for some $x$, and so $Q_{+} \neq \varnothing$, and we will discuss the distribution of the eigenvalues $\lambda^{i+}$ in $Q_{+}$. A similar discussion applies to the $\lambda^{i-} \in Q_{-}$if $Q_{-} \neq \varnothing$.

For any $\mathbf{u}=\left(u_{1}, \ldots, u_{k}\right) \in \bigoplus_{r=1}^{k} L^{2}(U)$ we let $W_{r}(\mathrm{u}), r=1, \ldots, k$, denote the matrix obtained by replacing the $r$ th row of $W(\mathbf{u})$ (defined in Section 5 ) with the vector $\omega=(1, \ldots, 1)$. Left definiteness implies that for each $r$ and each $\mathbf{u}$ with $u_{\mathrm{r}} \neq 0, r=1, \ldots, k$, $\operatorname{det} W_{r}(\mathbf{u})>0$, so the matrix $W_{r}(\mathbf{u})$ is non-singular.

Lemma 6.1. For any $v \in \omega^{\perp}$ there exist integers $r_{ \pm}$such that

$$
\pm v \cdot v_{r \pm}\left(x_{r}\right)>0, \quad \text { a.e. } \quad x_{r \pm} \in U
$$

Proof. The proof of this lemma is similar to the proof of Lemma 4.2, but using the matrices $W_{r}(\mathbf{u})$ instead of the matrices $V_{r}(\mathbf{x})$, as in the proof of Lemma 5.1.

Lemma 6.2. If the system (1.1), (1.2) is left definite then condition (3.5) holds.

Proof. The proof of this lemma is similar to the proof of Lemma 4.3, but using Lemma 6.1, rather than Lemma 4.2.

Corollary 6.3. If the system (1.1), (1.2) is left definite then $\left\|\lambda^{i+}\right\| \approx\|\mathbf{i}\|^{2}$, for all $\mathbf{i}$.

Thus, of all the definiteness conditions considered in this paper, only (non-uniform) right definiteness allows the eigenvalues to grow faster than $\|\mathbf{i}\|^{2}$. 
Lemma 6.4. Suppose that

$$
Z, \cap\left(N, \cup P_{r}\right) \cap \omega^{\perp} \subset\{0\} \cup \bigcup_{t=1}^{k}\left(\text { int } N_{t} \cup \text { int } P_{t}\right), \quad r=1, \ldots, k
$$

Then, for any $\varepsilon$ with $0<\varepsilon \leqq 1$, there is a number $\gamma(\varepsilon)>0$ such that for any $v \in \omega^{\perp}$, there are integers $r_{ \pm}$, for which

$$
\pm v \cdot v_{r}\left(x_{r \pm}\right) \geqq \gamma(\varepsilon)\|v\|, \quad x_{r \pm} \notin U_{r \pm}(v, \varepsilon),
$$

where the sets $U_{r \pm}(v, \varepsilon) \subset U$ have measures $\left|U_{r \pm}(v, \varepsilon)\right|<\varepsilon$.

Proof. The proof of this lemma is similar to the proof of Lemma 5.2.

Remark. When $k=2$, it follows from Lemma 6.1 that $Z_{r} \cap \omega^{\perp}=\{0\}$, so in this case condition (6.1) holds automatically.

We can now use Lemma 6.4 to obtain the following theorem on the distribution of the multiparameter eigenvalues in the left definite case, using similar methods to those used to obtain Theorems 4.6 and 5.4.

Theorem 6.5. If (6.1) holds then the mapping $\phi_{+}: Q_{+} \rightarrow \mathbf{R}_{+}^{k}$ is a homeomorphism, with inverse $\phi_{+}^{-1}: \mathbf{R}_{+}^{k} \rightarrow Q_{+}$. Also, for any $\varepsilon$ with $0<\varepsilon \leqq 1$, there is a number $\delta(\varepsilon)>0$ such that the restriction of $\phi_{+}^{-1}$ to $\bar{R}_{\varepsilon}^{k} \cap S_{1}$ is Lipschitz continuous with Lipschitz constant $\delta(\varepsilon)^{-1}$ and, for all $\mathbf{i} \in \overline{\mathbf{R}}_{\varepsilon}^{k}$,

$$
\lambda^{\mathrm{i}+}=\pi^{2} \phi_{+}^{-1}(\mathrm{i})+\delta(\varepsilon)^{-1} O(\|\mathrm{i}\|)=\pi^{2}\|\mathrm{i}\|^{2} \phi_{+}^{-1}(\mathrm{i} /\|\mathrm{i}\|)+\delta(\varepsilon)^{-1} O(\|\mathrm{i}\|) .
$$

\section{REFERENCES}

1. F. V. Atkinson, Discrete and Continuous Boundary Value Problems (Academic Press, New York, 1961).

2. P. Binding, Multiparameter definiteness conditions, Proc. Roy. Soc. Edinburgh 89A (1981), 319-332.

3. P. Binding, Dual variational approaches to multiparameter eigenvalue problems, J. Math. Anal. Appl. 92 (1983), 96-1 13.

4. P. Binding, Nonuniform right definiteness, J. Math. Anal. Appl. 102 (1984), 233-243.

5. P. Binding and H. Volkmer, Existence and uniqueness of indexed multiparametric eigenvalues. J. Math. Anal. Appl. 116 (1986), 131-146.

6. P. J. Browne and B. D. Sleeman, Asymptotic estimates for eigenvalues of right definite two parameter Sturm-Liouville problems, Proc. Edinburgh Math. Soc. 36 (1993), 391-397.

7. M. Falerman, On the distribution of the eigenvalues of a two-parameter system of ordinary differential equations of the second order, SIAM J. Math. Anal. 8 (1977), 854-870. 
8. M. Falerman, Distribution of eigenvalues of a two-parameter system of differential equations. Trans. Amer. Math. Soc. 247 (1979), 45-86.

9. E. INCE, Ordinary Differential Equations (Dover reprint, New York, 1956).

10. B. P. Rynne, The asymptotic distribution of the eigenvalues of right definite multiparameter Sturm-Liouville systems, Proc. Edinburgh Math. Soc. 36 (1993), 35-47.

11. B. D. Sleeman, Klein oscillation theorems for multiparameter eigenvalue problems in ordinary differential equations, Nieuw Arch. Wisk. 27 (1979), 341-362.

12. J. F. C. Kingman and S. J. TAYloR, Introduction to Measure and Probability (Cambridge University Press, 1966).

Department of Mathematics

Heriot.Watt University

RicCARTON

EDINBURGH EH14 4AS 